\title{
The effect of UCS intensity on the long-term retention of a classically conditioned fear response
}

\author{
MELVIN L. GOLDSTEIN \\ Indiana University, Kokomo, Indiana 46901
}

\begin{abstract}
An experiment was conducted to measure the effect of UCS intensity, in the classical fear conditioning situation, on long-term retention. White rats were conditioned at high $(2.63 \mathrm{~mA})$ and medium $(.83 \mathrm{~mA})$ UCS intensities and were tested for retention 2 weeks and 2 months later. The results of the experiment indicated that the magnitude of the classically conditioned fear response decreases 2 weeks after original conditioning, but that there is little further decrement after the original conditioning session. The original fear conditioning session produces a relatively stable level of fear that shows some dissipation, with time, at the medium UCS intensity level. Some fear remains, however, as long as 2 months after the original conditioning session.
\end{abstract}

McAllister and McAllister (1968) used the classical fear conditioning technique to show that there is a decrease in performance following a 47-h interval between condition and test sessions. A medium UCS intensity $(.83 \mathrm{~mA})$ was used. Miller and Kushel (cited in Miller, 1967) demonstrated that the effects of simple one-trial learning with electric shock were retained for as long as 23 days after conditioning. Kamin (1963) showed that a conditioned avoidance response was retained for as long as 20 days after conditioning. Goldstein (1974) found that the classically conditioned fear response was retained for as long as 1 week after conditioning with a high UCS intensity, but that there was no retention of the response $48 \mathrm{~h}$ after conditioning with a low UCS intensity.

The purpose of the present experiment was to extend these findings to longer retention intervals, such as 2 weeks and 2 months after classical fear conditioning. Rats were conditioned at high $(2.63 \mathrm{~mA})$ and medium $(.83 \mathrm{~mA})$ UCS intensities and were tested for retention 2 weeks and 2 months later.

\section{METHOD}

\section{Subjects}

The subjects were 10 naive male albino rats supplied by the Holtzman Company, Madison, Wisconsin. They were 90 days old on arrival and 120 to 150 days old at the start of the experiment. They were maintained, ad lib, on Purina Rat Chow throughout the course of the experiment.

There were five subjects in the high UCS-intensity group and five subjects in the medium UCS-intensity group.

\section{Apparatus}

The apparatus was a black conditioning box divided by a guillotine-type door and a hurdle into two compartments. The CS was a buzzer and light. The UCS was a 60 -cycle ac current conducted to the grid through a $250,000-0 h m$ series resistor.
UCS intensity was $.83 \mathrm{~mA}$ and $2.63 \mathrm{~mA}$. Further details of the apparatus have been described earlier (Goldstein, 1960, 1977).

\section{Procedure}

An experimental session was divided into "classical" conditioning and "instrumental" test sessions. There were six experimental sessions.

Phase 1. Exploration. Subject was placed into the grid compartment, with the guillotine door raised, for a period of $10 \mathrm{~min}$. CS and UCS were off, and the subject was permitted free exploration of both compartments of the conditioning box. At the end of the 10-min exploration period, the guillotine door was lowered and the subject was placed into the grid compartment for $1 \mathrm{~h}$ and $3 \mathrm{~min}$.

Phase 2. Classical conditioning. Classical conditioning trials were begun immediately after exploration and adaptation. A classical conditioning trial consisted of one successive presentation of CS and UCS. The interval between CS and UCS onset was $4 \mathrm{sec}$, UCS duration was $1 \mathrm{sec}$, and CS and UCS presentations were separated by 3 -min intervals. Subjects were given nine CS-UCS presentations.

Subjects were removed from the grid compartment $3 \mathrm{~min}$ after the start of the last conditioning trial and were taken to the home cage in the animal room, where they remained for $5 \mathrm{~min}$ with food and water available.

Phase 3. Exploration. A second 10-min exploration session in the conditioning box was given immediately afterward. At the end of this session, a final 10 -min period in the home cage was given.

Phase 4. Instrumental test trials. "Instrumental" hurdlejump conditioning sessions were started immediately after the last period in the home cage. The purpose of the hurdle-jump trials was to measure the strength of classical "fear" conditioning by presenting CS without UCS. Subjects were required to learn to jump across a hurdle from the grid floor into the woodenfloor compartment when CS was turned on and the door separating the two compartments was opened. CS was turned off as soon as the response had been made. The response measure used was hurdle-jump latency.

Each subject received 20 hurdle-jump acquisition trials during a 45-min test period that began $28 \mathrm{~min}$ after the last classical conditioning trial. These trials were separated by 2-min intervals.

Phase 5. Two-week retention test. A retention test was given 
2 weeks after original conditioning. The procedure was the same as for Phase 4.

Phase 6. Two-month retention test. A second retention test was given 2 months after the original conditioning session. The procedure used was the same as for Phase 5.

\section{RESULTS}

The hurdle-jump latencies were transformed to reciprocals and summed over blocks of five trials. The mean of a block of five trials was the score used in the statistical analysis.

The results for the original conditioning, 2-week retention, and 2-month retention sessions are shown in Figure 1.

A trend analysis of variance (Edwards, 1960, p. 228) was calculated for the results shown in Figure 1. The trial variable was significant beyond the .01 level $[F(11,66)=10.75]$. The Shock Level by Test Trials interaction was significant beyond the .01 level of confidence $[F(11,66)=3.00]$.

For the original conditioning session, the shock intensity variable was not significant $[F(1,6)=1.27]$. The test trial variable was significant beyond the .05 level of confidence $[F(3,18)=5.20]$. The Shock Level by Test Trials interaction was not significant $[F(3,18)=$ $.20]$.

For the 2-week retention session, the shock intensity variable was significant beyond the .05 level of confidence $[F(1,6)=6.43]$. The test trial variable was significant beyond the .01 level $[F(3,18)=8.5]$. The Shock Level by Test Trial interaction was significant beyond the .01 level $[F(3,18)=9.0]$.

For the 2-month retention test session, the shock intensity variable was short of significant at the .05 level of confidence $[F(1,6)=4.07]$. The test trial variable was significant beyond the .05 level. $[F(3,18)=$ 3.5]. The Shock Level by Test Trial interaction was not significant $[F(3,18)=.50]$.

A series of $t$ tests was calculated to test the significance of the session to session changes in performance shown in Figure 1. For the high-intensity UCS, the increase in performance between the last five trials of original conditioning and the last five trials of the 2-week retention session was not significant $[\mathrm{t}(6)=$ 1.47]. The drop in performance between the 2-week retention session and the 2-month retention session for the high-intensity UCS was short of significant at the .05 level $[\mathrm{t}(6)=1.90]$.

For the medium-intensity UCS, the drop in performance from the original conditioning session to the 2-week retention session was significant at the .05 level of confidence $[t(6)=3.00]$. The drop in performance from the 2-week retention session to the 2-month retention session was not significant $[\mathrm{t}(6)=1.29]$.



Figure 1. The effect of UCS intensity on 2-month and 2-week retention of a classically conditioned fear response.

\section{DISCUSSION}

The results of the present experiment indicate that the magnitude of the classically conditioned fear response decreases 2 weeks after original conditioning, but that there is little further decrement 2 months after the original conditioning session. The original fear conditioning session produces a relatively stable level of fear that shows some dissipation, with time, at the medium UCS-intensity level. Some fear remains, however, as long as 2 months after the original conditioning session.

The interpretation of this experiment assumes that the intensity of fear is linearly related to the strength of hurdlejump responding (McAllister \& McAllister, 1968). A recent study by Goldstein (1977) showed that strength of classical fear conditioning is linearly related to strength of hurdle-jump responding. The present study showed that the strength of hurdle-jump responding varied directly with the number of CS-UCS pairings and the intensity of UCS.

\section{REFERENCES}

EDWARDs, A. L. Experimental design in psychological research. New York: Holt, Rinehart, \& Winston, 1960.

Goldstein, M. L. Acquired drive strength as a joint function of shock intensity and number of acquisition trials. Journal of Experimental Psychology, 1960, 60, 349-358.

Goldstein, M. L. The effect of amygdalectomy on long-term retention of an undertrained classically conditioned fear response. Bulletin of the Psychonomic Society, 1974, 4, 548-550.

Goldstein, M. L. Acquired drive strength as a joint function of UCS intensity and number of CS-UCS pairings. Journal of General Psychology, 1977, 96, 5-61.

KAMIN, L. J. Retention of an incompletely learned avoidance response: Some further analysis. Journal of Comparative and Physiological Psychology, 1963, 56, 713-718.

McAllister, D. E., \& McAllister, W. R. Forgetting of acquired fear. Journal of Comparative and Physiological Psychology, 1968, 65, 352-355.

Miller, N. E. Laws of learning relevant to its biological basis. Proceedings of the American Philosophical Society, 1967, 111, 315-325.

(Received for publication March 1, 1979.) 